\title{
The role of external beam and endoluminal radiation boosting in rectal cancer
}

\author{
Maaike Berbée*,1, An-Sofie Verrijssen¹, Jeroen Buijsen ${ }^{1}$, Frank Verhaegen ${ }^{1}$ \& Evert Jan \\ Van Limbergen ${ }^{1}$ \\ ${ }^{1}$ Department of Radiation Oncology (MAASTRO), GROW School for Oncology \& Developmental Biology, Maastricht University \\ Medical Center, Doctor Tanslaan 12, 6229 ET Maastricht, The Netherlands \\ *Author for correspondence: maaike.berbee@maastro.nl
}

\section{"Different techniques have been used in experimental protocols to achieve radiation dose escalation."}

First draft submitted: 10 April 2019; Accepted for publication: 10 May 2019; Published online: 10 June 2019

Keywords: boost $\bullet$ brachytherapy $\bullet$ dose escalation $\bullet$ radiotherapy $\bullet$ rectal cancer

To date, standard care of rectal cancer includes surgical resection of the tumor. Depending on gross tumor characteristics, patients may receive neoadjuvant (chemo)radiotherapy in order to reduce the locoregional recurrence rate. With the currently used radiation dose of $45-50 \mathrm{~Gy}$, about $10-20 \%$ of all patients will develop a complete response after (chemo)radiotherapy. The number of patients with a complete response after (chemo)radiotherapy is likely to increase if higher radiation doses to the tumor would be used [1].

As dose escalation, although associated with more complete responses (CR), does not seem to result in improved locoregional control or survival when followed by total mesorectal excision surgery, dose escalation techniques are currently not part of standard practice [2]. Nonetheless, renewed interest currently exists in dose escalation, as in the case of a clinical CR it appears to be safe to offer patients follow-up instead of surgery, the so-called watch and wait strategy [3]. Surgery-related morbidity and side effects, for example a colostomy, can then be avoided. Different techniques have been used in experimental protocols to achieve radiation dose escalation. Both external beam radiotherapy (EBRT) and/or endoluminal techniques such as brachytherapy or contact x-ray radiotherapy (CXRT) have been attempted.

\section{Discussion}

The optimal treatment techniques still need to be determined. Various technical improvements are currently being developed and implemented regarding both external beam and endoluminal rectal boosting. To be successful, the techniques will have to result in higher complete response rates, limited acute and late toxicity and long-term adequate rectal function. Boosting will only be of added value to the patient if the added toxicity is very limited and it does not negatively affect quality of life in the long term.

The risk of radiation-induced toxicity depends both on the radiation dose to which normal tissue is exposed as well as on the volume of normal tissue that is irradiated [4]. Hence, in order to limit the risk of rectal toxicity in rectal boosting, the volume of healthy tissue exposed to the boost dose should be as small as possible. Moreover, the more selective the technique, the higher the radiation doses that can be applied to the tumor with an isotoxic effect, and the higher the expected CR rate.

\section{External beam radiotherapy}

Regarding the various potential techniques, an important advantage of external beam boosting is the fact that EBRT is available in all radiotherapy centers. It is the most commonly used radiotherapy method. However, as the tumor is being irradiated from the outside as opposed to from a short distance as in endoluminal techniques, external beam boosting is probably the least selective technique by nature. Moreover, treatment selectivity might 
be negatively affected by tumor movement due to changes in rectal and bladder filling and by poor visibility of the tumor on standard set-up imaging modalities such as cone beam CT.

Until now, the clinical results of external beam based rectal dose escalation have not been truly convincing [5-12]. Though feasibility has been shown in a number of series, the added value of the external boost with respect to an increased CR rate has not been unequivocally shown. The fact that no or disputable effects on improved CR rates have been reported until now, could very well be due to the modest dose increase that has been used. Boost doses in the range of 45-60 Gy have been applied. Considering the currently known dose-response curves, no major effect regarding CR rates should be expected at these dose levels. To enable dose escalation to higher levels, technical improvements are needed. The introduction of MR linac-based EBRT or the use of fiducials may enable more selective treatment and thereby higher levels of dose escalation. Moreover, optimizing the timing of the boost may improve results. From a theoretical perspective both the introduction of a simultaneously integrated boost technique as well as a sequential boost after standard treatment could be beneficial. By integrating the boost, the dose and damage to the surrounding tissues can be kept at a lower level. However, larger treatment margins might be needed in order to compensate for positional uncertainties. The potential advantage of a sequential boost after standard (chemo)radiotherapy is that the boost volume may be smaller due to tumor shrinkage [13]. To combine the advantages of both options, it has been suggested to apply the boost as an adaptive simultaneously integrated boost to the shrinking tumor during the second half of long course (chemo)radiotherapy [9,14,15]. During the coming years, it will become clear whether external beam based dose escalation with improved techniques and higher radiation doses does indeed result in significantly increased CR rates.

\section{Endoluminal radiotherapy}

Alternatively, important potential advantages of endoluminal techniques include the possibility to apply an even more selective/localized boost than when using EBRT.

The results of trials investigating the potential effect of rectal brachytherapy using high dose rate (HDR) radioactive sources are very promising. For example, Appelt et al. showed that an additional brachytherapy boost of 5 Gy after 60 Gy EBRT results in a clinical CR in $78 \%$ of patients with a resectable cT2-3N0 cancer [16]. These complete responders were not operated on but observed. About $15 \%$ of these patients developed a local regrowth. Rectal bleeding was the most common late toxicity, with 7\% G3 rectal bleeding 1 year after treatment. Another informative study is the Phase I HERBERT trial [17]. In this dose escalation trial in inoperable and elderly patients, EBRT ( $13 \times 3$ Gy) was followed by 3 weekly brachytherapy applications with increasing dose levels (5-8 Gy). The maximum tolerated fractional dose was set at $7 \mathrm{~Gy}$. Overall, a CR rate of $61 \%$ was observed. G3 late rectal toxicity, in the trial population as a whole, was seen in $40 \%$ of the patients. Hence, the results of these trials are extremely promising. However, considering the reported toxicity data, there is still a need for optimization of the methods.

Though general brachytherapy equipment is available in most radiotherapy centers, rectal brachytherapy is sparsely offered. This is probably because the current rectal brachytherapy methods are relatively complex. Moreover, rectal brachytherapy with the now available applicators is less selective than CXRT.

In rectal CXRT a $50 \mathrm{kV}$ x-ray tube is used to irradiate the tumor from a short distance. Due to the sharp depth-dose fall off characteristics of $50 \mathrm{kV}$ x-rays, a very localized radiation treatment can be applied with this technique. CXRT according to the Papillon method is currently being used with good results in a limited number of clinics. Due to the physical characteristics of $50 \mathrm{kV}$ XCRT, rectal mucosal lesions can be treated with very high fraction doses up to $30 \mathrm{~Gy}$ and total doses up to 90 Gy without causing significant normal tissue toxicity. In selected patients, CR rates up to $63 \%$ have been reported [18]. Although the reported results are excellent, widespread introduction of the technique remains limited because of the availability of the equipment. Moreover, no randomized studies are available using CXRT with CR as a primary end point. Hence, the results from the currently recruiting randomized Phase III OPERA trial are awaited. In this trial, the safety and efficacy of a CXRTand EBRT-based boost are being compared.

In order to overcome the problem of the limited availability of CXRT equipment, novel brachytherapy applicators are under development. The introduction of, for example, the FLOWER applicator, an HDR brachytherapy applicator that allows irradiation with the same dose profile as in CXRT, may increase the availability of CXRT-like therapies [19]. An additional potential advantage of the FLOWER applicator is the possibility of accurate dose calculation and individualized treatment planning.

It needs to be noted that not only technical improvements are needed. To improve outcome, a better understanding of tumor biology is crucial to determine: the optimal time point for a radiation boost, the treatment margins 
needed to cover all viable microscopic tumor spread and to develop tools to select patients who will benefit from a boost.

Furthermore, to enable adequate evaluation of the various techniques and potential improvements, it is important that not only CR rates, but also toxicity data are being reported systematically in relation to the irradiated volumes. Hence, consensus guidelines on dose reporting and treatment volume specification should be implemented as soon as possible. This will allow a comparison of the various techniques and accurate modeling of tumor control and normal tissue toxicity probabilities (TCP/NTPC) [20].

\section{Conclusion}

In conclusion, it is expected that dose escalation with either EBRT or endoluminal radiation techniques will increase the complete response rate, and thereby the chance of organ preservation, in rectal cancer patients. Successful implementation of such a strategy requires adequate tools for patient selection and a radiation technique which results in a high complete response rate in combination with limited toxicity and good long-term rectal function and quality of life.

\section{Financial \& competing interests disclosure}

BM, VF and EJVL obtained patent for an endorectal probe device for effecting radiation treatment of colorectal cancerous tissue in the rectum of a human or animal subject pending to Maastricht University. The authors have no other relevant affiliations or financial involvement with any organization or entity with a financial interest in or financial conflict with the subject matter or materials discussed in the manuscript apart from those disclosed.

No writing assistance was utilized in the production of this manuscript.

\section{Open access}

This work is licensed under the Attribution-NonCommercial-NoDerivatives 4.0 Unported License. To view a copy of this license, visit http://creativecommons.org/licenses/by-nc-nd/4.0/

\section{References}

1. Appelt AL, Ploen J, Vogelius IR, Bentzen SM, Jakobsen A. Radiation dose-response model for locally advanced rectal cancer after preoperative chemoradiation therapy. Int. J. Radiat. Oncol. Biol. Phys. 85(1), 74-80 (2013).

2. Appelt AL, Vogelius IR, Ploen J et al. Long-term results of a randomized trial in locally advanced rectal cancer: no benefit from adding a brachytherapy boost. Int. J. Radiat. Oncol. Biol. Phys. 90(1), 110-118 (2014).

3. Van Der Valk MJM, Hilling DE, Bastiaannet $\mathrm{E}$ et al. Long-term outcomes of clinical complete responders after neoadjuvant treatment for rectal cancer in the International Watch \& Wait Database (IWWD): an international multicentre registry study. Lancet 391(10139), 2537-2545 (2018).

4. Fonteyne V, Ost P, Vanpachtenbeke F et al. Rectal toxicity after intensity modulated radiotherapy for prostate cancer: which rectal dose volume constraints should we use? Radiother. Oncol. 113(3), 398-403 (2014).

5. But-Hadzic J, Anderluh F, Brecelj E et al. Acute toxicity and tumor response in locally advanced rectal cancer after preoperative chemoradiation therapy with shortening of the overall treatment time using intensity-modulated radiation therapy with simultaneous integrated boost: a Phase II trial. Int. J. Radiat. Oncol. Biol. Phys. 96(5), 1003-1010 (2016).

6. Janjan NA, Crane CN, Feig BW et al. Prospective trial of preoperative concomitant boost radiotherapy with continuous infusion 5-fluorouracil for locally advanced rectal cancer. Int. J. Radiat. Oncol. Biol. Phys. 47(3), 713-718 (2000).

7. Lee JH, Kim DY, Nam TK et al. Long-term follow-up of preoperative pelvic radiation therapy and concomitant boost irradiation in locally advanced rectal cancer patients: a multi-institutional Phase II study (KROG 04-01). Int. J. Radiat. Oncol. Biol. Phys. 84(4), 955-961 (2012).

8. Lupattelli M, Matrone F, Gambacorta MA et al. Preoperative intensity-modulated radiotherapy with a simultaneous integrated boost combined with Capecitabine in locally advanced rectal cancer: short-term results of a multicentric study. Radiat. Oncol. 12(1), 139 (2017).

9. Passoni P, Fiorino C, Slim N et al. Feasibility of an adaptive strategy in preoperative radiochemotherapy for rectal cancer with image-guided tomotherapy: boosting the dose to the shrinking tumor. Int. J. Radiat. Oncol. Biol. Phys. 87(1), 67-72 (2013).

10. Bae BK, Kang MK, Kim JC et al. Simultaneous integrated boost intensity-modulated radiotherapy versus 3-dimensional conformal radiotherapy in preoperative concurrent chemoradiotherapy for locally advanced rectal cancer. Radiat. Oncol. J. 35(3), 208-216 (2017).

11. Alongi F, Fersino S, Mazzola R et al. Radiation dose intensification in pre-operative chemo-radiotherapy for locally advanced rectal cancer. Clin. Transl. Oncol. 19(2), 189-196 (2017). 
12. Wiltshire KL, Ward IG, Swallow C et al. Preoperative radiation with concurrent chemotherapy for resectable rectal cancer: effect of dose escalation on pathologic complete response, local recurrence-free survival, disease-free survival, and overall survival. Int. J. Radiat. Oncol. Biol. Phys. 64(3), 709-716 (2006).

13. Van Den Begin R, Kleijnen JP, Engels B et al. Tumor volume regression during preoperative chemoradiotherapy for rectal cancer: a prospective observational study with weekly MRI. Acta Oncol. 57(6), 723-727 (2018).

14. Maggiulli E, Fiorino C, Passoni $\mathrm{P}$ et al. Characterisation of rectal motion during neo-adjuvant radiochemotherapy for rectal cancer with image-guided tomotherapy: implications for adaptive dose escalation strategies. Acta Oncol. 51(3), 318-324 (2012).

15. Raso R, Scalco E, Fiorino C et al. Assessment and clinical validation of margins for adaptive simultaneous integrated boost in neo-adjuvant radiochemotherapy for rectal cancer. Phys. Med. 31(2), 167-172 (2015).

16. Appelt AL, Ploen J, Harling $\mathrm{H}$ et al. High-dose chemoradiotherapy and watchful waiting for distal rectal cancer: a prospective observational study. Lancet Oncol. 16(8), 919-927 (2015).

17. Rijkmans EC, Cats A, Nout RA et al. Endorectal brachytherapy boost after external beam radiation therapy in elderly or medically inoperable patients with rectal cancer: primary outcomes of the Phase I HERBERT study. Int. J. Radiat. Oncol. Biol. Phys. 98(4), 908-917 (2017).

18. Sun Myint A, Smith FM, Gollins S et al. Dose escalation using contact x-ray brachytherapy after external beam radiotherapy as nonsurgical treatment option for rectal cancer: outcomes from a single-center experience. Int. J. Radiat. Oncol. Biol. Phys. 100(3), 565-573 (2018).

19. Bellezzo M, Fonseca GP, Verrijssen AS et al. A novel rectal applicator for contact radiotherapy with HDR (192)Ir sources. Brachytherapy 17(6), 1037-1044 (2018).

20. Verrijssen AS, Opbroek T, Bellezzo M et al. A systematic review comparing radiation toxicity after various endorectal techniques. Brachytherapy 18(1), 71-86, e75 (2019). 